\title{
Radar-coding and Geocoding Look Up Tables for the Fusion of GIS Data and SAR images in Mountain Areas
}

Ivan Pétillot, Emmanuel Trouvé, Philippe Bolon, Andreea Julea

Université de Savoie, Polytech'Savoie/LISTIC, 74944 Annecy-le-Vieux, France

Michel Gay, Jean-Michel Vanpé

GIPSA-lab, CNRS - INP Grenoble, France

\begin{abstract}
For merging Synthetic Aperture Radar (SAR) with georeferenced data, one usually uses processing software to orthorectify the SAR images in order to add them in a GIS. Nevertheless, this projection involves an important alteration of the information of SAR images and are often specific to some particular SAR sources. Moreover, the transformation of radarcoding data from a GIS into the radar geometry allows the use of different information sources to improve the analysis of SAR images and the result interpretation. In this paper, we propose a simple and efficient method to build two couples of Look Up Tables (LUT) allowing to code data in ground or radar geometry. This approach is applied to a high relief area in the Alps, where satellite and airborne SAR images are used for glacier evolution monitoring.
\end{abstract}

\section{Introduction}

High relief areas present a difficult context for data fusion : optical images are dependent on the presence of clouds and snow and radar images are distorted by foldover and shadow due to the relief [1]. These irreversible degradations affect the data which have to be rectified in order to be combined either in optical geometry or in radar geometry. Some processing softwares, such as those dedicated to differential SAR interferometry (D-InSAR), include geocoding tools which allow to resample SAR data in ground geometry. The inverse operation consists in projecting georeferenced data such as optical images or ground measurements in each specific radar geometry. To perform it for SAR images in mountainous areas is not straightforward since the two geometries are not linked by a bijective transformation. Specific tools, which takes SAR sensor geometry into account, are required. Very few softwares offer the end-user with convenient tools to perform this task.

In this paper, a convenient and generic approach is proposed to perform direct and inverse geocoding for the use of SAR images in high relief areas. This approach is based on the simulation of the geometric component of the backscattered signal, followed by the correlation with the real SAR image and the computation two sets of look- 
up tables (LUT) which respectively provides the position of georeferenced points in the radar geometry, and the latitude longitude coordinates of each SAR pixel. Our main contribution consists in proposing a complete method which is usable with both space-borne and airborne SAR data, and requires only a georeferenced Digital Terrain Model (DTM) and the trajectory of the aircraft or satellite. The performances of the method are assessed and different applications illustrate the use of the results in the context of Alpine glacier monitoring.

\section{Simulation of SAR image}

The processing chain of the proposed method is shown in Figure 1. Based on the radar parameters and the available DTM, four steps are necessary to obtain the LUT. First, a ground mask is computed, consisting of a map of visible, foldover and shadow points. Then a simulation of the ground radar intensity is performed and resampled in the slant range geometry. Finally, the result is compared to the real SAR image in order to improve the geometric parameters of the simulation and to obtain the final registration which provides the sought-after LUT. Two couples of LUT are produced :

- One couple to "radar-rectify" a georeferenced data in the specific geometry of the SAR image. These LUT are matched on the radar image dimensions and contains the ground coordinates of each pixels in radar.

- One couple to geocode a SAR data. These LUT are georeferenced and allow to know, for each ground point, where is located its equivalent in the specific radar geometry of the SAR image.

\subsection{Data preprocessing}

For simulating the radar image, three methods are described in [4]. One of them, the "incoherent simulation", requires only the knowledge of the local incidence angles to process the pixel values of the final SAR image. This geometric approach is chosen for its convenience in mountain areas where relief has the main influence on the backscattered intensity of the radar image [5].

At first, a DTM in latitude and longitude coordinates is used. The DTM is rotated and resampled in order to bring lines parallele to the lines of a SAR acquisition and columns correspond to the azimuth direction.

We consider that the radar wave is spherical. Computations of localizations and distances are performed in the three dimensional WGS-84 ellipsoide referential.

[Figure 1 about here.]

\subsection{Visibility mask}

The specific radar imaging geometry [6], based on range measurements, leads to geometric distortions in the resulting image, such as foldover and shadow.

[Figure 2 about here.] 
Active foldover occurs if the terrain slope exceeds the radar viewing angle [7] and active shadow if the local incidence angle exceeds $90^{\circ}$. Passive foldover is induced by the vicinity of corresponding active zones [8].

In mountain areas, radar shadow or foldover make large areas of the received signal useless for operational purposes. For SAR image and interferogram interpretation, the accurate prediction of such areas is very useful [9].

Using the DTM, we determine the so called visible areas which are not distorted by irreversible geometric distortions.

\subsection{Ground range processing}

To simulate a SAR image, the backscattered radar intensity on ground range has to be modelled. SAR simulation system is a wide topic with various applications [2], [3]. Some efficient and realistic solutions have been proposed using three dimensional textured models of objects to be simulated [10] but in alpine environment, the topographical effects have a stronger influence than thematic ones. Here a cosine backscatter model is sufficiently realistic to simulate the radar intensity [11] for the sought-after application : a final registration of the simulation with the real image.

\subsection{Slant range processing}

Two different approaches can be used to bring images from the ground range to a geometry specific to the SAR sensor (slant range).

- The inverse approach where each pixel of each line of the slant image is inspected for searching its correspondence in the ground image. For each line of the ground image, an ordered vector is created containing the distances from the points on the ground surface to the satellite. It determines the neighbors of each slant pixel which value is then computed according to these neighbors. The way this value is computed depends on the type of the image to be resampled in the SAR geometry.

- The second method is named direct approach by intervals. In this case, pixels intervals on the groud range are projected on the slant range in order to obtain a complete covering of the slant axis.

One important step of the simulation is to obtain the synthetic image of the backscattered radar intensity in slant range. This synthetic image will allow to compare the results with real SAR images in order to improve the LUT accuracy. The so called direct approach by intervals method is used to obtain the intensity in the radar geometry. In each slant pixel every ground point of the interval projected gives a contribution. To derive the corresponding intensity in slant range, an integration is applied within the slant resolution cell of the ground intensities weighted by square cardinal sine function in order to take into account the distance to the centre of the cell. We limited ourselves to the principal lobe of the sinc function and the rectangles method is used to calculate the integral.

\subsection{LUT computation}

The next step of the method consists in computing LUT which describe the transformations between radar and ground geometries. In other words, to build up maps of correspondences allowing to find the georeferencement of each point of 
the SAR image, and, for each ground point, where it is located in the SAR image.

On one hand, as the Digital Terrain Model (DTM) used is georeferenced, the radar-coding LUT can be represented by a pair of images with range and azimuth axis, which provides in each point its latitude and longitude coordinates. These LUT are obtained by an inverse approach which projects maps of latitude and longitude coordinates of the DTM points. According to the distances between ground points and the satellite, a new value is assigned to each slant pixel.

On the second hand, the geocoding LUT are estimated from the distances between ground points and the satellite because the SAR image pixels are sampled on these distances in the line of sight direction.

\subsection{Final RST refining}

[Figure 3 about here.]

The simulated image is then compared to the real SAR image. A correlation-based matching of sub-windows is applied to find out the final geometric transformation necessary to register the simulated image to the real one. The results can be used either directly on the simulated image, or to improve the accuracy on the simulation parameters and iterate the simulation. In both cases, the shifts between the simulation and the real image provide a RST mapping (Rotation Scale Translation). In the case of a direct use on simulation, it leads to smaller degradations than that produced by a polynomial transformation. In the case of a refining of the initial parameters, the various inaccuracies are corrected and the simulation processed again. The corrections concern the initial rotation of the DTM, the sampling rate in azimuth direction and the slant rate revealing errors on the initial emission angle.

\section{Results and discussion}

Results are illustrated over the Chamonix Mont-Blanc test site on spaceborne images (ERS, ENVISAT and ALOS data) and airborne E-SAR images. The test site is located in the Alps, near the borders between France, Italy and Switzerland.

\subsection{Performance assessment}

In order to evaluate the method accuracy, two data sets are used : one spaceborne and one airborne SAR data. The first one is a low resolution ERS image acquired in march 1996 and covering the Chamonix valley region. The second one is a high resolution E-SAR image from the DLR acquired in February 2007 and covering the Argentière glacier.

Three tests are done by radar-coding an image, then geocoding it and last comparing this image to the original one :

- a performance assessment of the LUT from ground to SAR by using the ERS data set and geocoding LUT generated by the ROI_PAC (Repeat Orbit Interferometry Package) software.

- a performance assessment of the LUT from SAR to ground and more precisely the coherence between the LUT from SAR to ground and the LUT from ground to SAR, using the E-SAR data set. 
- finally a test on the preservation of the altimetry on both data sets.

For the first performance assessment, simple images of the $\mathrm{X}$-axis and Y-axis coordinates are transformed in the specific geometry of the ERS datum. This radar-rectification is performed by three ways : directly with a polynomial transformation, with our LUT by using the final RST matching to refine parameters and with our LUT with a final RST transformation. The geocoding LUT generated by the ROI_PAC software [11] are used as a "ground truth" to back-tansform to ground the two radar-coded images. Table 1 presents the shifts between the resulting images and the original ones over an area including Argentière and Mer-De-Glace glaciers. It can be noticed that the method using a final RST matching is the most accurate for rectifying images in the radar geometry in a high relief area like the Mont-Blanc region.

[Table 1 about here.]

For the second performance assessment, the two simple test images are rectified in the specific geometry of the E-SAR image. A DTM of the region and the trajectory of the airplane are used to perform the simulation and to generate both geocoding and radar-coding LUT. The DTM has been rotated by 52 degres to correspond to the trajectory orientation of the airplane and its resolution has been resampled from 10x10 metres to 2.5x3 metres for the registration with the SAR data after the radar-coding $(\simeq 3.6 \times 3$ metres). The final RST matching gives an error on the rotation of 2 degres and scale factors of 0.967 in the slant range and 1.003 in the azimuth direction. The test images are radar-coded and geocoded thanks to the two couples of LUT and the divergences with the original test images over the visible part of the Argentière glacier are reported in Table 2. The area where the differences are processed covers $6 \mathrm{~km}^{2}$ (672 648 pixels), with altitudes from 1295 metres to 2884 metres. The results show good correspondings between the two couples of LUT. $98 \%$ of distances between the two images are inferior to 0.5 pixels, and distances greater than 0.5 pixels are due to points very near from a foldover region induced from the high relief of the glacier edges .

[Table 2 about here.]

The third test concerns the preservation of the altimetry. In the spaceborne data set (low resolution), $75 \%$ of points have a variation of their altitude less than 15 metres. Compared to the altitude resolution of the DTM and the steep slopes of this high mountains region, this result is acceptable for the processing of any low resolution data. In the airborne data set (high resolution), the differences mean is -0.0065 metres with a standard deviation of 0.262 metres. $98,3 \%$ of pixels have a difference of altitudes after the radar-coding and the geocoding transformations less than 0.26 metres.

\subsection{Applications for processing purpose}

The first results of the processing chain are the visibility masks necessary to the simulation. They can be used to realise visibility predictions about future acquisitions in order to determine which are the best configurations. Results are presented in the region covering the Argentière and Mer-De-Glace glaciers for ALOS Figure 4 and ENVISAT Figure 5 satellites.

[Figure 4 about here.] 
[Figure 5 about here.]

Figure 6-(a) presents the simulated SAR intensity image in a flight direction along the Argentière glacier. This image is then matched to the real E-SAR (October 2006, L band) intensity image Figure 6-(b) to process LUT. The main advantage of the proposed method is that, once the slant range projection of the LUT is processed, additional information can be radar coded and made available in the D-InSAR processing chain. For example, in the differential interferogram computation, the topographic phase component must be compensated for obtaining the displacement along the radar line of sight. This can be done by rewrapping the radar coded altitude and subtracting it from the initial interferogram [12]. Another application is the use of ground truth for supervised classifications. For exemple, a supervised classification of the E-SAR image is performed for detecting crevasses on the Argentière glacier. First, a radar coded orthophotography of the glacier (see Figure 6-(c)) is used for determining crevasses and non-crevasses training regions. Figure 6-d shows the training regions (red for the crevasses region and blue for the non-crevasses one), represented in the Pauli decomposition [13] of the E-SAR image. They are used to initialise a K-mean supervised classification with the wishart distance. At each iteration, cluster centres are estimated and pixels are affected to the nearest class, according to the complex Wishart distribution [14]. Ten iterations of the algorithm are done to obtain the result presented in Figure 6-e. The radar rectified orthophotography is then used again to check the validity of the resulting classification image, where we can actually extract the two main areas of crevasses and other small crevasses spread over the glacier.

[Figure 6 about here.]

\subsection{Applications for visual analysis}

Another way to use the proposed LUT is to link directly images in different geometries through a viewing software (see Figure 7). This method enables to preserve the integrity of both georeferenced images and radar data, since no geometrical processing is applied to them. For example, a geographic map, or other georeferenced data can be used to guide the radar image interpretation without any resampling.

In figure 7, we use an IGN scan covered by the shadow and layover map at right to locate some points or areas of the interferometric ERS image (10-11 march 1996, Chamonix Valley) at left. The geographic coordinates and elevation are also obtained for any interesting points of the radar image. Thus we can extract displacement fringes from the interferometric phase corresponding to small glaciers appearing in the georeferenced ground map. We avoid therefore to use mistaken areas like foldover or non-displacement fringes from static areas bordering on glaciers.

[Figure 7 about here.]

\section{Conclusions}

In this paper, a new method aiming at facilitating the processing of different information sources which include SAR images in the difficult context of alpine glacier monitoring is introduced. The proposed method consists in using a DTM 
to detect foldover and shadow areas, to simulate SAR intensity images in both ground and radar geometry, and finally to process LUT that allow to combine georeferenced data (such as optical images, slopes, land use maps) and radar measurements (such as strong scatterers, coherence and displacement fields). The radar-coding approach is much more interesting than geocoding to understand the physical phenomena occurring in SAR images or to provide prior information useful for the SAR interferometric processing whereas the geocoding approach allows to merge data to use GIS softwares. These two approaches have been explored by processing two couples of LUT allowing to code data in SAR or ground geometry without having to execute the processing chain again.

The accuracy of the obtained LUT mainly depends on the DTM resolution. That is an unavoidable restriction to the use of the latest high resolution SAR data. Moreover the geometric accuracy is restricted by the naive approach : some acquisition configuration parameters like the exact location of the area simulated in the final real image, or the ground texture, are not taken into account. Even if the simulation of the SAR intensity is done just for improving the LUT precision by registering it on the real SAR data, the geometric accuracy of the simulation facilitate the final correlationbased matching. However, by using only a small number of parameters and a three dimensional model based on the WGS-84 ellipsoid, the proposed approach has the advantage of being generic and operational for both space-borne and airborne SAR data in the different parts of the world. Since the simulation does not introduce any information regarding the backscattering targets, the same look-up tables can be applied for the different polarimetric channels. The main limitation is due to the hypothesis that the backscattering coefficient is dominated by the topographic effects. This is valid in the case of strong relief which creates bright features corresponding to the foldover areas. However, in flat areas where this hypothesis does not hold, the SAR geometric distortion are more limited and conventional geocoding tools are often sufficient.

Further work will consist in extending this approach to merge images from different SAR sources over Alpine glaciers in order to extract spatio-temporal information using either change detection methods developed for SAR images and optical data or data mining techniques. An open-source "beta version" of the software associated to the proposed approach and the Graphic User Interface illustrated in Figure 7 are available (www.efidir.fr) and will benefit from the user feedback.

Acknowledgment The authors wish to thank the French Research Agency (ANR) for supporting this work through the MEGATOR (ACI-MD-04, www.lis.inpg.fr/megator) and EFIDIR project (ANR-2007-MDC0-04, www.efidir.fr). The authors wish to thank to the European Space Agency for the provision of ERS SAR data through the Earth Observation Announcement of Opportunity (Category 1 proposal 3525) just as the RGD (Régie de Gestion Données 73-74) for their useful data in the Mont-Blanc region. They would like to express their gratitude to the Microwaves and Radar Institute DLR-HR for the E-SAR images and all participants of the experiment performed in October 2006 and February 2007. 


\section{References}

[1] W. G. Kropatsch and D. Strobl. The generation of SAR layover and shadow maps from digital elevation models. IEEE Transactions on Geoscience and Remote Sensing, 28(1):98-107, January 1990.

[2] F. Xu and Y.-Q. Jin. Imaging simulation of polarimetric SAR for a comprehensive terrain scene using the mapping and projection algorithm. IEEE Transactions on Geoscience and Remote Sensing, 44(11):3219-3234, November 2006.

[3] G. Margarit. Marine applications of SAR polarimetry. PhD thesis, 2007. University of Catalunya.

[4] F.W. Leberl. Radargrammetric Image Processing. Artech House Remote Sensing Library, Norwood, 1990.

[5] B. Guindon. Development of a SAR data acquisition planning tool (SARPLAN) based on image simulation. International Journal of Remote Sensing, 14(2):333-344, 1993.

[6] G. Schreier. SAR Geocoding: Data and Systems, chapter Geometrical properties of SAR images, pages 103-134. Karlsruhe, Germany, Wichmann, 1993.

[7] R. Kwok, J. C. Curlander, and S. S. Pang. Rectification of terrain induced distortions in radar imagery. Photogrammetric Engineering and Remote Sensing, 53(5):507-513, May 1987.

[8] M. Gelautz, H. Frick, J. Raggam, J. Burgstaller, and F. Leberl. SAR image simulation and analysis of alpine terrain. ISPRS Journal of Photogrammetry and Remote Sensing, 53(1):17-38, 1998.

[9] M. Eineder. Efficient Simulation of SAR Interferograms of Large Areas and of Rugged Terrain . IEEE Transactions on Geoscience and Remote Sensing, 41(6):1415-1427, 2003.

[10] C. Cochin, P. Pouliguen, B. Delahaye, D. Le Hellard, P. Gosselin, and F. Aubineau. MOCEM - An 'all in one' tool to simulate SAR image. In European Conference on Synthetic Aperture Radar (EUSAR-2008), CD-ROM, Graf-Zeppelin-Haus, Friedrichshafen, Germany, volume 1, page 4 pages, 2008 .

[11] S. Buckley. ROI_PAC Documentation - Repeat Orbit Interferometry Package. PhD thesis, 2000. abriged version of chapter 3 of PhD thesis.

[12] D. Massonnet and K. Feigl. Radar interferometry and its application to changes in the Earth's surface. Reviews of Geophysics, 36(4):441-500, 1998.

[13] S. R. Cloude and K. P. Papathanassiou. Polarimetric SAR interferometry. IEEE Transactions on Geoscience and Remote Sensing, 36(5):15511565,1998

[14] J. S. Lee, M. R. Grunes, T. L. Ainsworth, D. Li-Jen, D. L. Schuler, and S. R. Cloude. Unsupervised classification using polarimetric decomposition and the complex Wishart classifier. IEEE Transactions on Geoscience and Remote Sensing, 37(5):2249-2258, 1999. 


\section{List of Figures}

1 processing chain to build the LUT allowing to radar rectify georeferenced data and to geocode SAR image. 10

2 Radar distortions of the slant range resampling due to the relief. . . . . . . . . . . . . . 11

3 Final RST matching to improve the LUT accuracy. The final transformation is applied on the images of

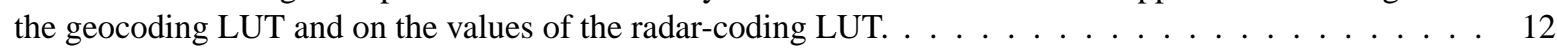

4 Visibility masks comparison of ALOS satellite acquisitions over Argentière and Mer-De-Glace glaciers. . 13

5 Visibility percentages comparison of ENVISAT satellite acquisitions over the Chamonix valley. . . . . 14

6 Argentière glacier in Chamonix valley : (a) simulated intensity in slant range, (b) real SAR intensity, (c) orthophotography in slant range, (d) Pauli decomposition with the two training regions, (e) classification

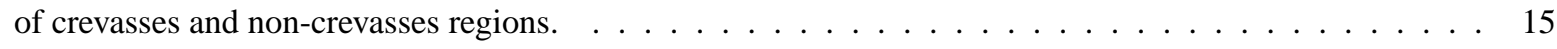

7 display of two stacks of data (radar geometry at left and georeferenced at right) linked thanks to the

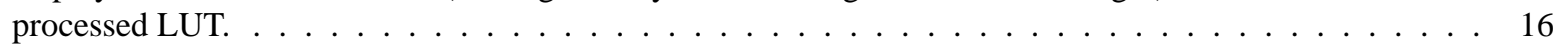




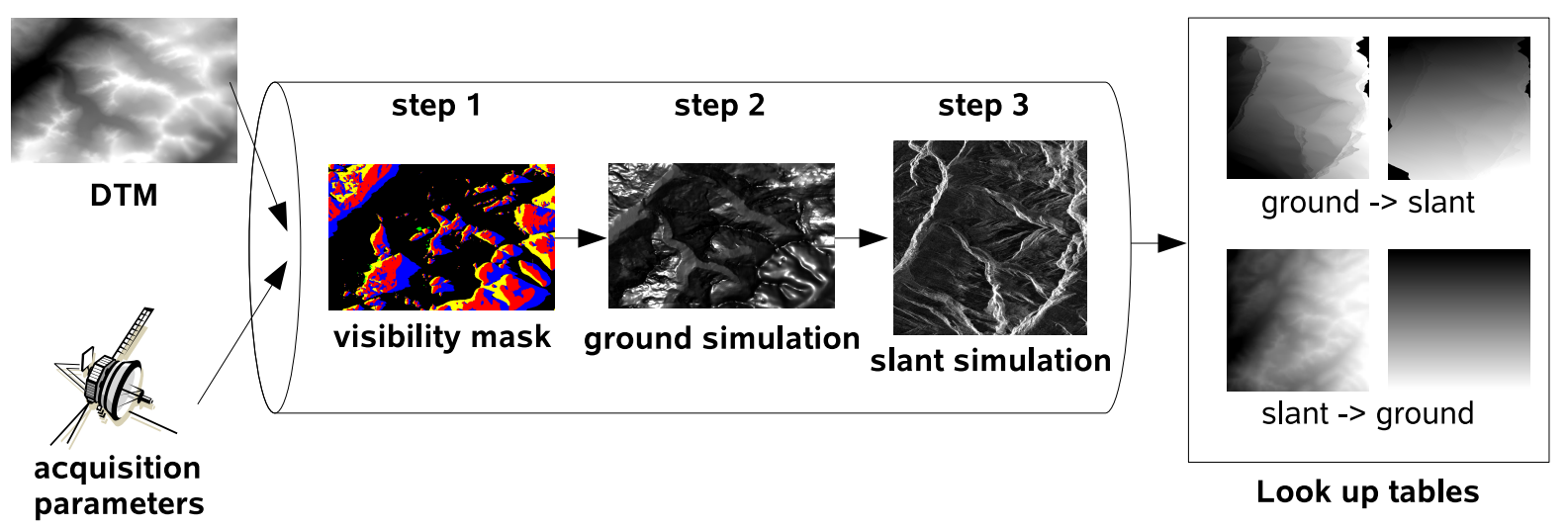

Figure 1: processing chain to build the LUT allowing to radar rectify georeferenced data and to geocode SAR image. 


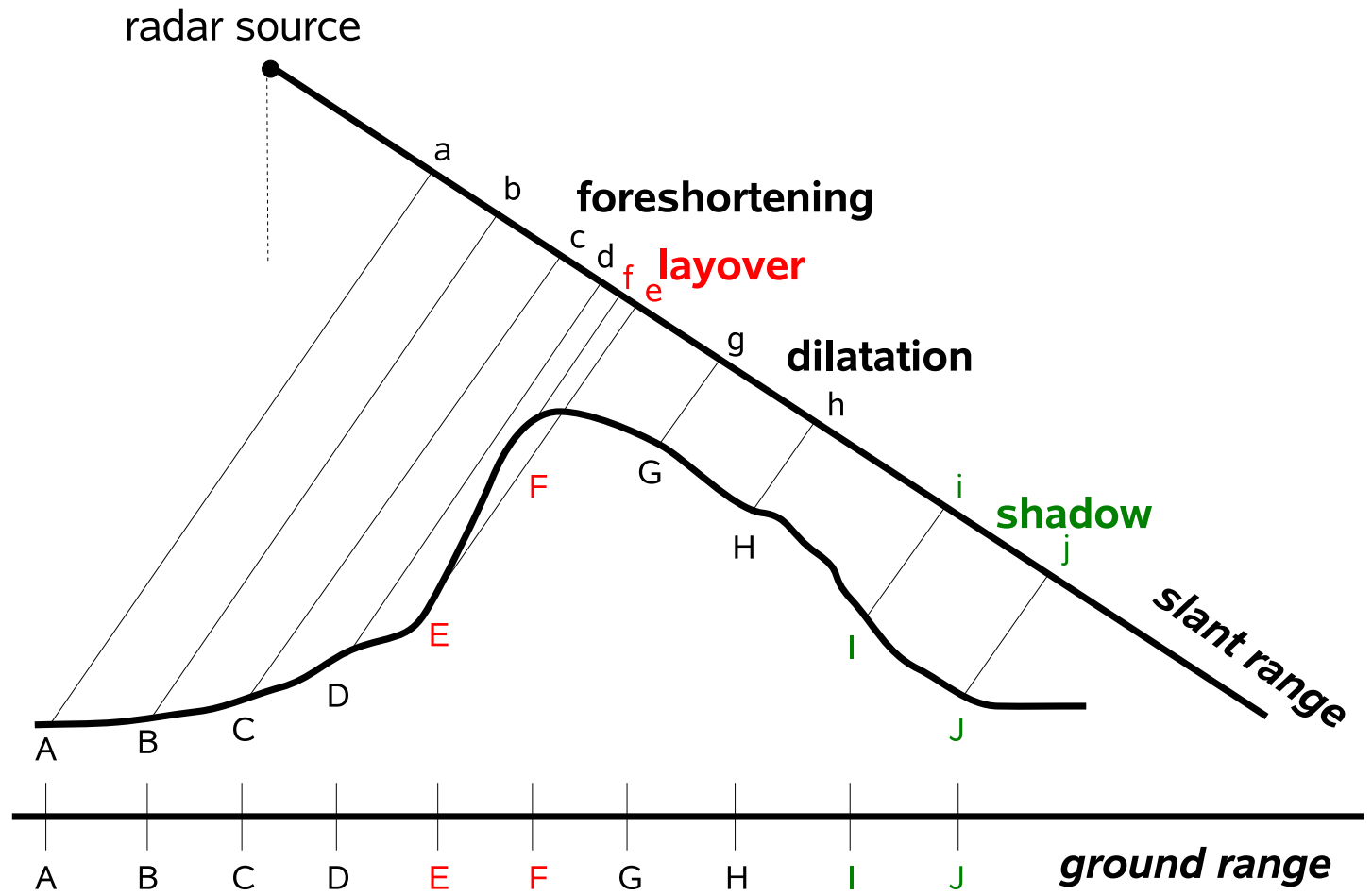

Figure 2: Radar distortions of the slant range resampling due to the relief. 


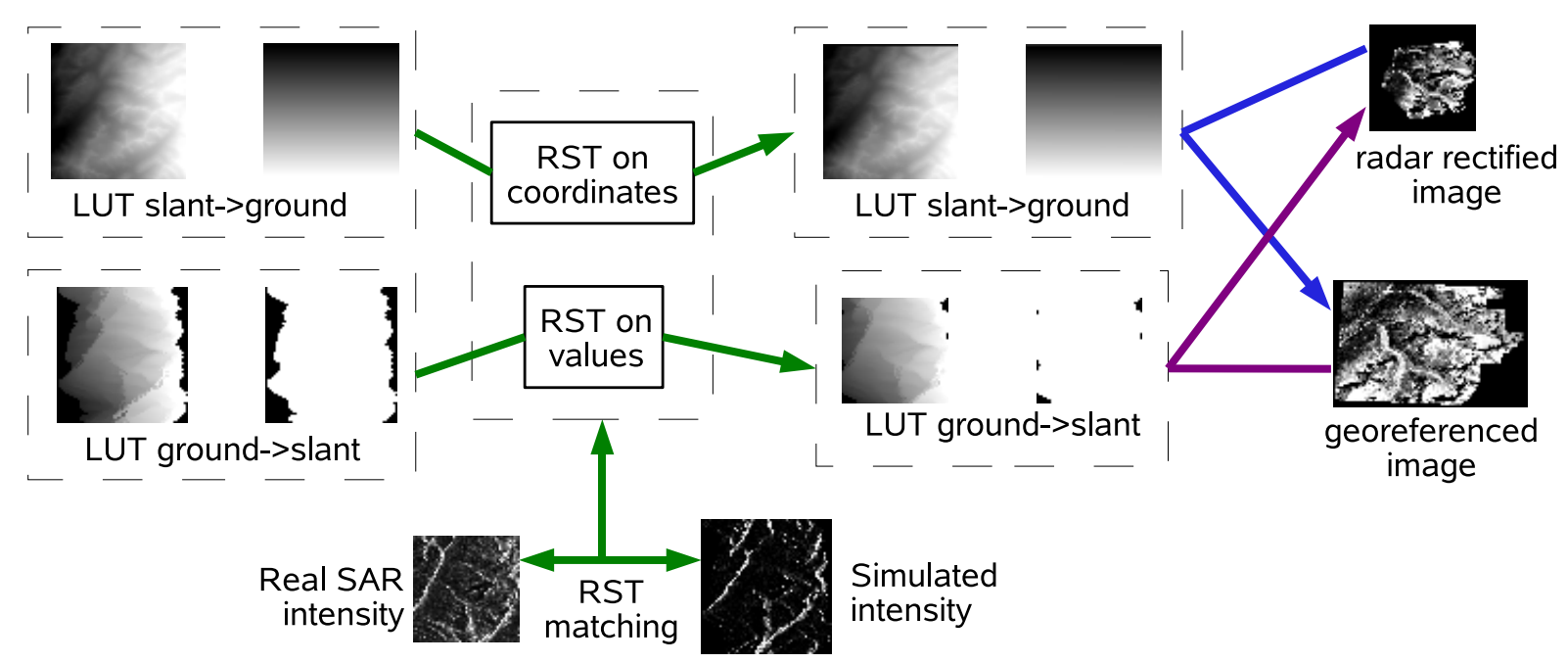

Figure 3: Final RST matching to improve the LUT accuracy. The final transformation is applied on the images of the geocoding LUT and on the values of the radar-coding LUT. 


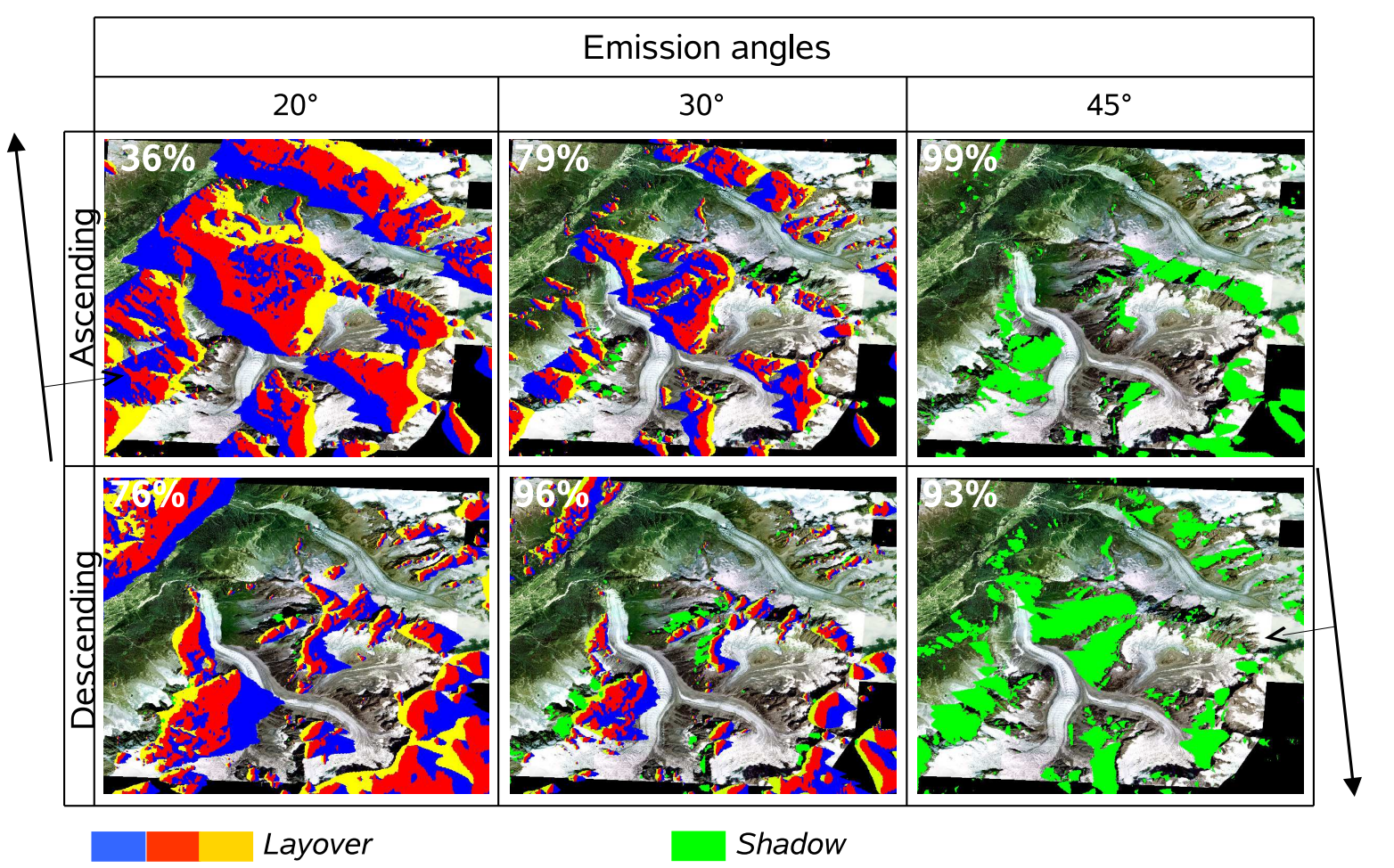

Figure 4: Visibility masks comparison of ALOS satellite acquisitions over Argentière and Mer-De-Glace glaciers. 

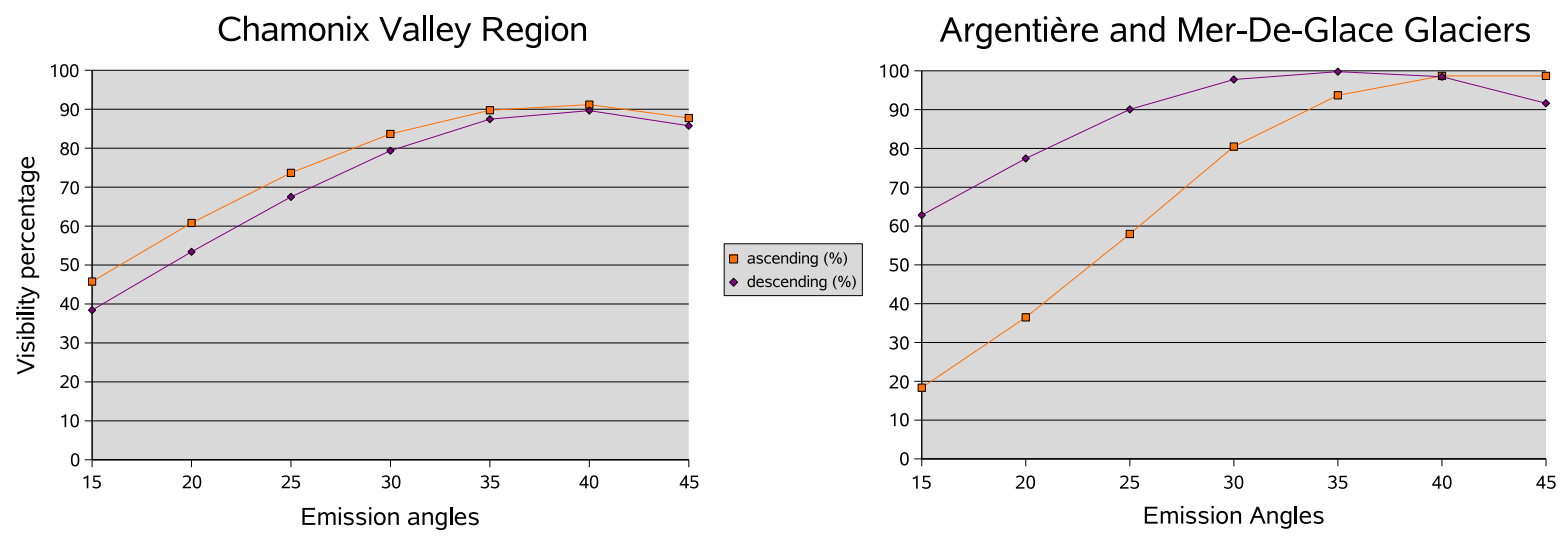

Figure 5: Visibility percentages comparison of ENVISAT satellite acquisitions over the Chamonix valley. 
(a)

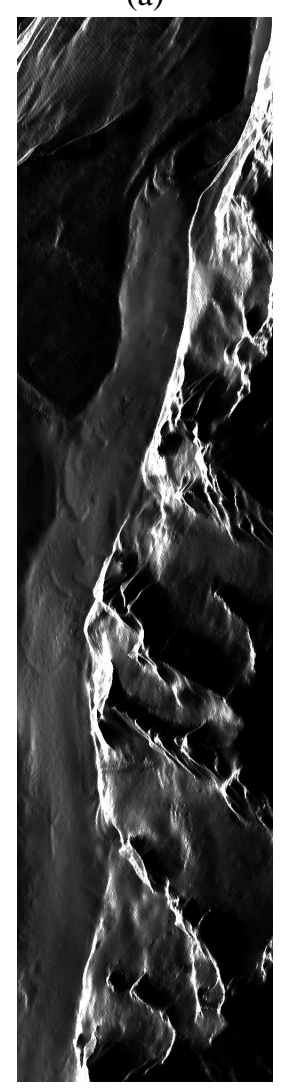

(b)

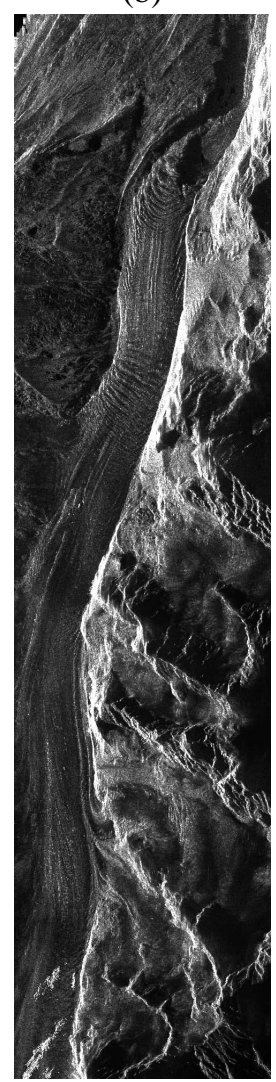

(c)

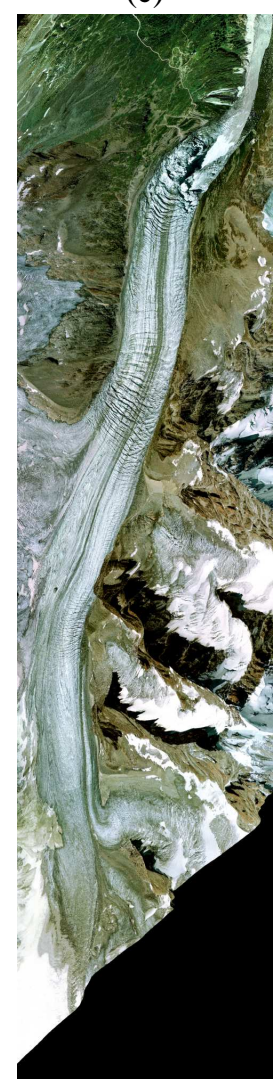

(d)

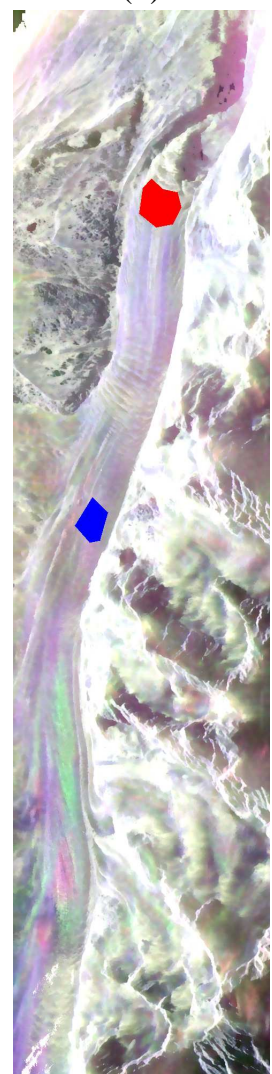

(e)

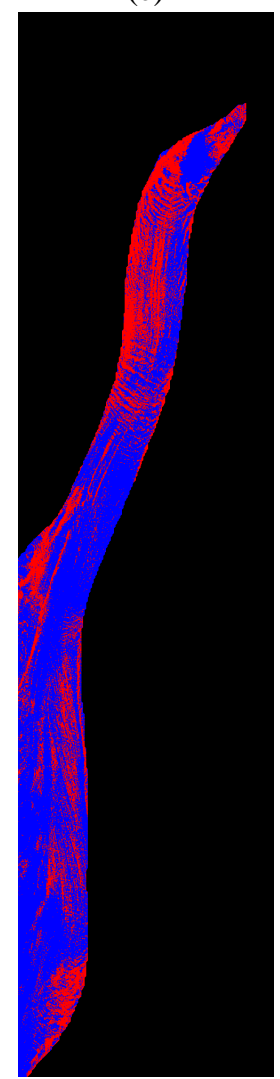

Figure 6: Argentière glacier in Chamonix valley : (a) simulated intensity in slant range, (b) real SAR intensity, (c) orthophotography in slant range, (d) Pauli decomposition with the two training regions, (e) classification of crevasses and non-crevasses regions. 


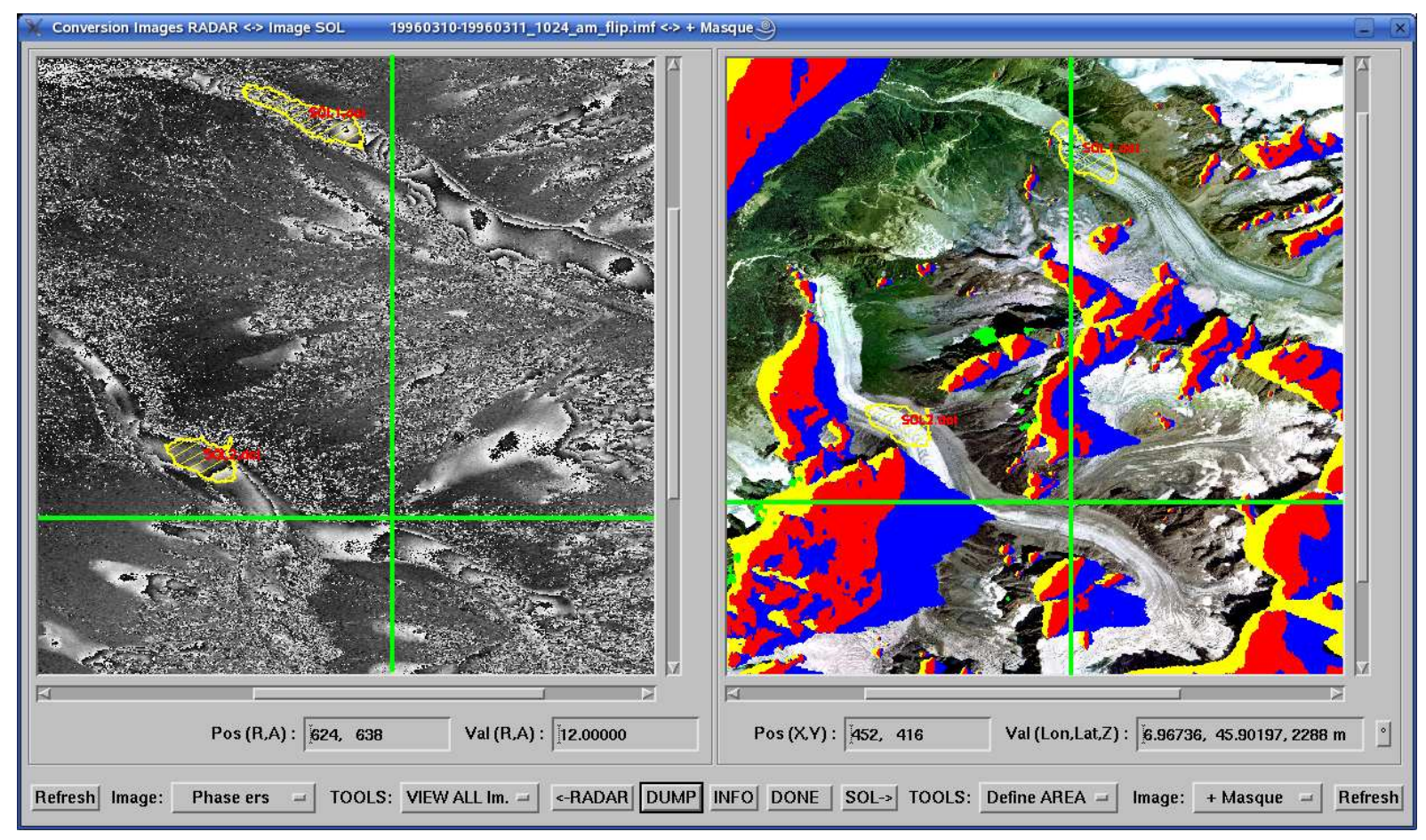

Figure 7: display of two stacks of data (radar geometry at left and georeferenced at right) linked thanks to the processed LUT. 


\section{List of Tables}

1 Means and standard deviations in pixels $(\simeq 16$ metres) between the test images before and after the two transformations. Distances are $\sqrt{\left(i-i^{\prime}\right)^{2}+\left(j-j^{\prime}\right)^{2}} \ldots \ldots \ldots \ldots \ldots \ldots$

2 Means and standard deviations in pixels ( $\simeq 3$ metres) between the test images before and after the two

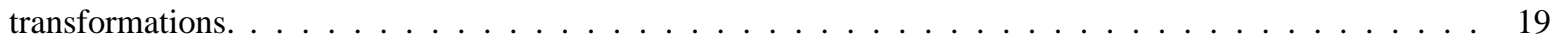




\begin{tabular}{|l|l|l|l|}
\hline IMAGES & $\begin{array}{l}\text { DIRECT POLYNOMIAL } \\
\text { TRANSFORMATION }\end{array}$ & $\begin{array}{l}\text { LUT + PARAMETERS } \\
\text { REFINING }\end{array}$ & $\begin{array}{l}\text { LUT + RST } \\
\text { REFINING }\end{array}$ \\
\hline \hline X-Axis & 85.54 & -2.37 & 1.58 \\
$\left(j-j^{\prime}\right)$ & $+/-40.03$ & $+/-13.21$ & $+/-1.57$ \\
\hline Y-Axis & -5.86 & -4.92 & -2.29 \\
$\left(i-i^{\prime}\right)$ & $+/-2.86$ & $+/-8.57$ & $+/-1.35$ \\
\hline distances & 85.71 & 14.27 & 3.17 \\
& $+/-40.06$ & $+/-8.61$ & $+/-1.77$ \\
\hline
\end{tabular}

Table 1: Means and standard deviations in pixels ( $\simeq 16$ metres) between the test images before and after the two transformations. Distances are $\sqrt{\left(i-i^{\prime}\right)^{2}+\left(j-j^{\prime}\right)^{2}}$. 


\begin{tabular}{|l|l|l|}
\hline IMAGES & $\begin{array}{l}\text { LUT WITHOUT SAR } \\
\text { REGISTRATION }\end{array}$ & $\begin{array}{l}\text { LUT + RST } \\
\text { REGISTRATION }\end{array}$ \\
\hline \hline X-Axis & -0.002 & -0.001 \\
$\left(j-j^{\prime}\right)$ & $+/-0.96$ & $+/-1.002$ \\
\hline Y-Axis & 0.00006 & 0.001 \\
$\left(i-i^{\prime}\right)$ & $+/-0.021$ & $+/-0.019$ \\
\hline distances & 0.098 & 0.14 \\
$\sqrt{\left(i-i^{\prime}\right)^{2}+\left(j-j^{\prime}\right)^{2}}$ & $+/-0.95$ & $+/-0.99$ \\
\hline angles & -0.36 & -0.19 \\
$\arctan \left(\frac{j-j^{\prime}}{i-i^{\prime}}\right)$ & $+/-1.14$ & $+/-1.30$ \\
\hline
\end{tabular}

Table 2: Means and standard deviations in pixels ( $\simeq 3$ metres) between the test images before and after the two transformations. 